\title{
Radiative Contributions Dominate Plasmon Broadening for Post-Transition Metals in the Ultraviolet
}

\author{
Maria V. Fonseca Guzman, Michael B. Ross* \\ Department of Chemistry, University of Massachusetts Lowell, Lowell, MA 01854
}

Keywords: Plasmonics, nanoparticles, UV, photonics, linewidth, post-transition metal

\begin{abstract}
We use classical electrodynamics calculations to investigate the plasmonic properties of the post-transition metals $\mathrm{Al}, \mathrm{Bi}, \mathrm{Ga}$, In, and $\mathrm{Sn}$, which are active in the ultraviolet, focusing in particular on the material- and resonance-dependent origins of plasmon broadening. Analytic Mie theory, the modified-long wavelength approximation, and the quasistatic dipole approximation together show that radiative processes dominate plasmon dephasing and damping in small (5-25 $\mathrm{nm}$ radius) $\mathrm{Al}, \mathrm{Bi}, \mathrm{Ga}, \mathrm{In}$, and $\mathrm{Sn}$ spheres. For $\mathrm{Al}, \mathrm{Ga}, \mathrm{In}$, and $\mathrm{Sn}$, the radiative contribution $(\sim 0.1-$ $0.2 \mathrm{eV})$ to the plasmon linewidth is 100 -fold greater than the non-radiative contribution $(0.001-$ $0.02 \mathrm{eV}$ ) derived from the bulk dielectric function. This is significantly different than what is observed for $\mathrm{Ag}$ spheres, where non-radiative contributions $(\sim 0.1 \mathrm{eV})$ are the primary source of broadening up to a radius of $25 \mathrm{~nm}$. Overall, these data suggest that the plasmonic properties, dephasing, and lifetimes for $\mathrm{Al}, \mathrm{Ga}$, In, and $\mathrm{Sn}$ - and to a lesser extent $\mathrm{Bi}$ - spheres are qualitatively similar. To develop a more general understanding of the relationship between
\end{abstract}


plasmon energy and linewidth, we use a model for ideal free-electron Drude metals. It is seen that the linewidth increases at higher energies even for lossless Drude metals, suggesting that the increased broadening observed in UV-active metals is a generalizable observation. These data have important implications for the use of these metals for ultraviolet plasmonics. The increased importance of radiative damping for post-transition metals could influence the ability to harvest photons, generate hot carriers, and enhance spectroscopy in the ultraviolet while providing new opportunities for manipulating high-energy photons.

\section{Introduction}

Plasmonic metal nanoparticles have the remarkable ability to confine light into small volumes. This phenomenon is due to localized surface plasmon resonance (LSPR), the collective oscillation of conduction electrons. ${ }^{1-3}$ As a consequence of the LSPR, plasmonic nanoparticles strongly absorb and scatter light and enhance electric fields at their surface. These properties have proven useful for applications in sensing, Raman spectroscopy enhancement, metamaterials, and catalysis. $^{4-8}$

The majority of work in this field has focused on Ag and Au nanoparticles because they exhibit strong LSPRs in the visible and NIR regions and are easy to synthesize. ${ }^{9}$ Recent efforts have expanded plasmonic capabilities into the UV and mid-infrared regions by focusing on a broader set of plasmonic materials. ${ }^{10-12}$ The UV region is of particular interest because it presents opportunities for high-energy photon harvesting, ${ }^{5}$ strongly enhanced spectroscopies due to resonance in organic molecules and polymers, ${ }^{4,}{ }^{13} \mathrm{UV}$ lithography, ${ }^{14}$ and enhanced optical interactions with DNA and proteins. ${ }^{15-18}$ 
Continued progress in this area requires understanding the fundamental and materialdependent behavior of UV-active plasmonic metals. Numerous studies have investigated the underlying photonic, electronic, and material-dependent mechanisms that determine the intensity and quality of the LSPR for Ag and Au. ${ }^{10-11,19-21}$ There is a less well-developed understanding of these mechanisms in the subset of UV-active plasmonic metals, including $\mathrm{Al}, \mathrm{Bi}, \mathrm{Ga}, \mathrm{In}, \mathrm{Mg}, \mathrm{Rh}$, and Sn. ${ }^{12,22-29}$ Recent work suggests that Al nanoparticles have plasmonic behavior that is distinct from that typically seen for the noble metals, such that there are differences in the plasmon linewidth, dephasing, and lifetime. ${ }^{21,30-34}$ Previous calculations for Bi nanoparticles support the potential design of a plasmonic metamaterial with switchable optical filtering applications in the near UV region. ${ }^{35}$ Better understanding of the material-dependent plasmonic properties for the broader set of potential UV-plasmonic metals is important for their continued development and application.

Analysis of the LSPR linewidth is particularly useful for understanding the materialdependent plasmonic response. Using the plasmon linewidth, one can investigate the complex dephasing processes that describe how the LSPR undergoes decay via electron-phonon interactions. ${ }^{20-21,36}$ While the linewidths are well-studied in $\mathrm{Au}$ and $\mathrm{Ag},{ }^{18-20}$ and to a lesser extent, $\mathrm{Al}^{21,}{ }^{29-33}$ the material-dependent contributions to the LSPR for promising UV active metals, such as $\mathrm{Bi}, \mathrm{Ga}$, In, and $\mathrm{Sn}$, have not been systematically deconstructed. ${ }^{37-38}$ Doing so is essential for understanding which materials are most promising in the UV. Previous work has shown that the dephasing process is highly material dependent. ${ }^{21}$ In general, there are two contributions to the plasmon linewidth: radiative and non-radiative. Radiative contributions describe interactions between photons emitted by the particle and the polarization of electrons within the particle. Nonradiative contributions describe intrinsic electronic transitions determined by the composition that 
are derived from the bulk dielectric function. In noble metals, the plasmon quality and lifetime are dictated by a balanced combination of non-radiative and radiative contributions for most particle sizes. ${ }^{20-21}$ For Al, however, it was shown that radiative broadening dominates the LSPR in small spherical particles active in the $200-250 \mathrm{~nm}$ region. ${ }^{21,31}$ Understanding the contributions to the linewidth—specifically, the balance of radiative and non-radiative contributions — is essential for designing UV plasmonic materials with efficient LSPRs, long plasmon lifetimes, and strongly enhanced near fields.

In this Article, we explore the contributions to the plasmon linewidth in $\mathrm{Ag}, \mathrm{Al}, \mathrm{Bi}, \mathrm{Ga}, \mathrm{In}$, and Sn spheres. Using generalized Mie theory, the modified long-wavelength approximation (MLWA), and the quasistatic approximation, we explore the size- and material-dependent LSPR for these elements. Dielectric functions were taken from the following references: $\mathrm{Ag},{ }^{39} \mathrm{Al},{ }^{40}$ $\mathrm{Bi},{ }^{41} \mathrm{Ga},{ }^{42-43} \mathrm{In}^{44}$ and $\mathrm{Sn}^{45}$. We report that for small (5-25 nm radius) $\mathrm{Al}, \mathrm{Bi}, \mathrm{Ga}$, In and $\mathrm{Sn}$ spheres active in the UV, radiative contributions are the dominant broadening process for the LSPR. Specifically, radiative contributions account for $>95 \%$ of the LSPR linewidth for all spherical Al, $\mathrm{Ga}$, In, and Sn sizes considered (5-25 nm); this is in contrast to Ag nanoparticles of the same size, where non-radiative contributions are the primary determinant of LSPR broadening. Consequently, we find the quasistatic approximation cannot accurately describe small $\mathrm{Al}, \mathrm{Bi}, \mathrm{Ga}$, In, and Sn spheres due to the importance of field retardation effects for all sizes. MLWA, meanwhile, accurately describes the dipolar LSPR in spheres up to a radius of $\sim 25 \mathrm{~nm}$ due to its ability to account for radiative damping and dynamic depolarization. For radii greater than $\sim 25$ $\mathrm{nm}$, Mie theory is required to account for multipolar resonances. We quantify the plasmonic quality factor and lifetimes for these elements and find that the post-transition metals $\mathrm{Al}, \mathrm{Ga}, \mathrm{In}$, and $\mathrm{Sn}$ have similar plasmonic behavior. When taken together, these data suggest that $\mathrm{Al}, \mathrm{Bi}, \mathrm{Ga}$, 
In, and Sn have distinct LSPRs from Ag and Au due to the increased importance of radiative broadening and the relatively small non-radiative contributions in the UV. Using an ideal freeelectron Drude model, we find that plasmon linewidth increases for lossless high plasma frequency metals. Furthermore, the differences in the plasmonic behavior between the post-transition metals can be understood in relation to their Drude-like behavior, the location of the LSPR, and the intrinsic dielectric function of the metal. This has important implications for local field enhancement, the importance of relative absorption to scattering, and the ability to utilize hot carriers. $^{4,6,11-12,46}$

\section{Materials and Methods}

\section{Generalized Mie Theory}

Mie theory calculations were used to calculate the optical response of spherical metal nanoparticles with a radius of 5-40 nm, 25 vector spherical harmonics were included for all calculations. ${ }^{3}{ }^{47}$ Extinction, absorption, and scattering contributions are reported as optical efficiency, Q, which is a unitless quantity. Surface scattering was not accounted for because it was found to have a relatively small effect in previous work for $\mathrm{Al},{ }^{31}$ and a background dielectric constant of 1 (vacuum) was used for all calculations. Mie theory allows the calculation of the extinction cross-section for a sphere, which is made up of the absorption and scattering contributions (Equations 1-3). ${ }^{3,48}$

$$
C_{e x t}=\frac{2 \pi}{k^{2}} \sum_{n=1}^{\infty}(2 n+1) \operatorname{Re}\left(a_{n}+b_{n}\right)
$$




$$
C_{\text {sca }}=\frac{2 \pi}{k^{2}} \sum_{n=1}^{\infty}(2 n+1)\left(\left|a_{n}\right|^{2}+\left|b_{n}\right|^{2}\right)
$$

$$
\mathrm{C}_{\mathrm{abs}}=\mathrm{C}_{\mathrm{ext}}-\mathrm{C}_{\mathrm{sca}}
$$

Where $k$ is the wavevector given by $k=2 \pi \varepsilon_{b}^{1 / 2} / \lambda . a_{n}$ and $b_{n}$ are the scattering coefficients which contain the Riccati-Bessel functions used in Mie theory. ${ }^{48-49}$ We report extinction, absorption, and scattering as efficiency, which is equal to the ratio of the cross-section to the particle geometric cross-section, $\pi a^{2}$.

Quasistatic Dipole Approximation

For spheres that are small relative to the wavelength, the quasistatic dipole approximation can describe the LSPR. ${ }^{1}$ The induced dipole is given by

$$
\mathbf{P}=\alpha \mathbf{E}(4)
$$

Where $\mathbf{E}$ is the incident electromagnetic field and the polarizability of a sphere is given by

$$
\alpha=4 \pi \varepsilon_{b} a^{3} \frac{\varepsilon-\varepsilon_{b}}{\varepsilon+2 \varepsilon_{b}}(5)
$$

Where $\varepsilon$ is the complex frequency-dependent dielectric function, $\varepsilon_{\mathrm{b}}$ is the dielectric constant for the background media, and $a$ is the radius of a sphere. The quasistatic solution for the cross-section of a sphere can be used to obtain the extinction cross-section given by Equation 6. ${ }^{1,49}$

$$
C_{\mathrm{ext}, \mathrm{qs}}=4 \pi k a^{3} \operatorname{Im}\left(\frac{\varepsilon-\varepsilon_{b}}{\varepsilon+2 \varepsilon_{b}}\right)
$$


Modified Long-Wavelength Approximation (MLWA)

For spheres that are large relative to the wavelength, ${ }^{1}$ the radiative corrected field $\mathbf{E}_{\text {rad }}$ must be accounted for in Equation 4, such that

$$
\mathbf{P}=\alpha\left[\mathbf{E}+\mathbf{E}_{\text {rad }}\right](7)
$$

Where $\mathbf{E}_{\text {rad is }}$ described by

$$
\mathbf{E}_{\mathrm{rad}}=\frac{2}{3} i k^{3} \mathbf{P}+\frac{k^{2}}{a} \mathbf{P}(8)
$$

In the medium $n$ where $k$ is the wavevector. The size-corrected dipole moment $\mathbf{P}$ can be determined by multiplying Equation 4 by

$$
g=\left[1-\frac{2}{3} i k^{3} \alpha-\frac{k^{2}}{a} \alpha\right]^{-1}
$$

Here, the radiation damping term scales by $\mathrm{k}^{3}$ and dynamic depolarization term scales by $\mathrm{k}^{2}{ }^{1}$

\section{Linewidth Fitting and Decomposition}

The total homogeneous linewidth $\Gamma$ is determined by fitting the dipolar extinction peak with a sum of Gaussian and Lorentzian functions, which helps account for peak asymmetry. This method works best for particles that do not exhibit higher-order resonances (e.g. quadrupoles), thus the values determined for the linewidths are limited to the particle radius at which a quadrupole is observed for each element.

The total linewidth is determined by the combination of the radiative and non-radiative contributions ${ }^{19-20,50}$

$$
\Gamma=\Gamma_{\text {radiative }}+\Gamma_{\text {non-radiative }}(10)
$$


where $\Gamma$ is the linewidth of the LSPR based on $Q_{\text {ext }}$, and $\Gamma_{\text {non-radiative }}$ is determined from the dielectric function according to $19-20,51$

$$
\Gamma_{\text {non-radiative }}=\frac{2 \operatorname{Im}\{\varepsilon\}}{\sqrt{(\partial \operatorname{Re}\{\varepsilon\} / \partial \omega)^{2}+(\partial \operatorname{Im}\{\varepsilon\} / \partial \omega)^{2}}}(11)
$$

where $\Gamma_{\text {non-radiative }}$ is the linewidth associated with a dielectric function, $\Gamma_{\text {radiative }}$ is determined from $\Gamma_{\text {radiative }}=\Gamma-\Gamma_{\text {non-radiative }}$. Equation 11 accounts for the bulk contributions to the linewidth derived from the dielectric function but does not account for surface scattering. ${ }^{19,51}$ This is excluded because surface interactions can be strongly dependent on the unique surface chemistry of a nanoparticle, which would differ significantly for the metals described herein. ${ }^{20-21,36}$ Additionally, previous related work on Al suggests that surface scattering effects are small in this size regime. ${ }^{31}$

\section{Ideal Drude-like Model}

The permittivity of an ideal free electron gas Drude metal was described using the DrudeSommerfeld model:2, 10, 52-54

$$
\varepsilon(\omega)=1-\frac{\omega_{p}^{2}}{\omega(\omega+i \gamma)}(12)
$$

where $\omega_{p}$ is the bulk plasma frequency and $\gamma$ is a phenomenological scattering rate. For the metals described herein, the scattering rate was set to 0.01 . The dielectric functions of the ideal freeelectron metals are calculated using a range of plasma frequencies from $6-20 \mathrm{eV}$. The dielectric function is used for calculating the extinction efficiency using Mie theory and linewidth fitting as described above. 


\section{Results and Discussion}

Optical Properties of Ag, $A l, B i, G a$, In, and Sn Spheres

Multiple levels of theory were used to investigate the optical properties of spheres with radii from 5-40 $\mathrm{nm}$. Spherical nanoparticles were chosen due to their high-energy LSPRs that can exist in the deep UV. Figure 1 depicts the Mie theory calculated size-dependent extinction efficiency for $\mathrm{Ag}, \mathrm{Al}, \mathrm{Bi}, \mathrm{Ga}, \mathrm{In}$, and $\mathrm{Sn}$ spheres. $\mathrm{Al}, \mathrm{Ga}$, In, and Sn support LSPRs well into the deep UV from 150-300 nm, Bi supports a broad LSPR 250-350 nm, and Ag supports an LSPR from $350-380 \mathrm{~nm}$. The higher energy onset of the LSPR is material-dependent and is determined by the dielectric function for each metal. While the analysis of the dielectric functions for these metals has been covered elsewhere, ${ }^{10-11,25}$ a comparison of the free electron density in these metals qualitatively illustrates why $\mathrm{Al}, \mathrm{Bi}, \mathrm{Ga}, \mathrm{In}$, and $\mathrm{Sn}$ can support higher energy plasmonic resonances than Ag. 

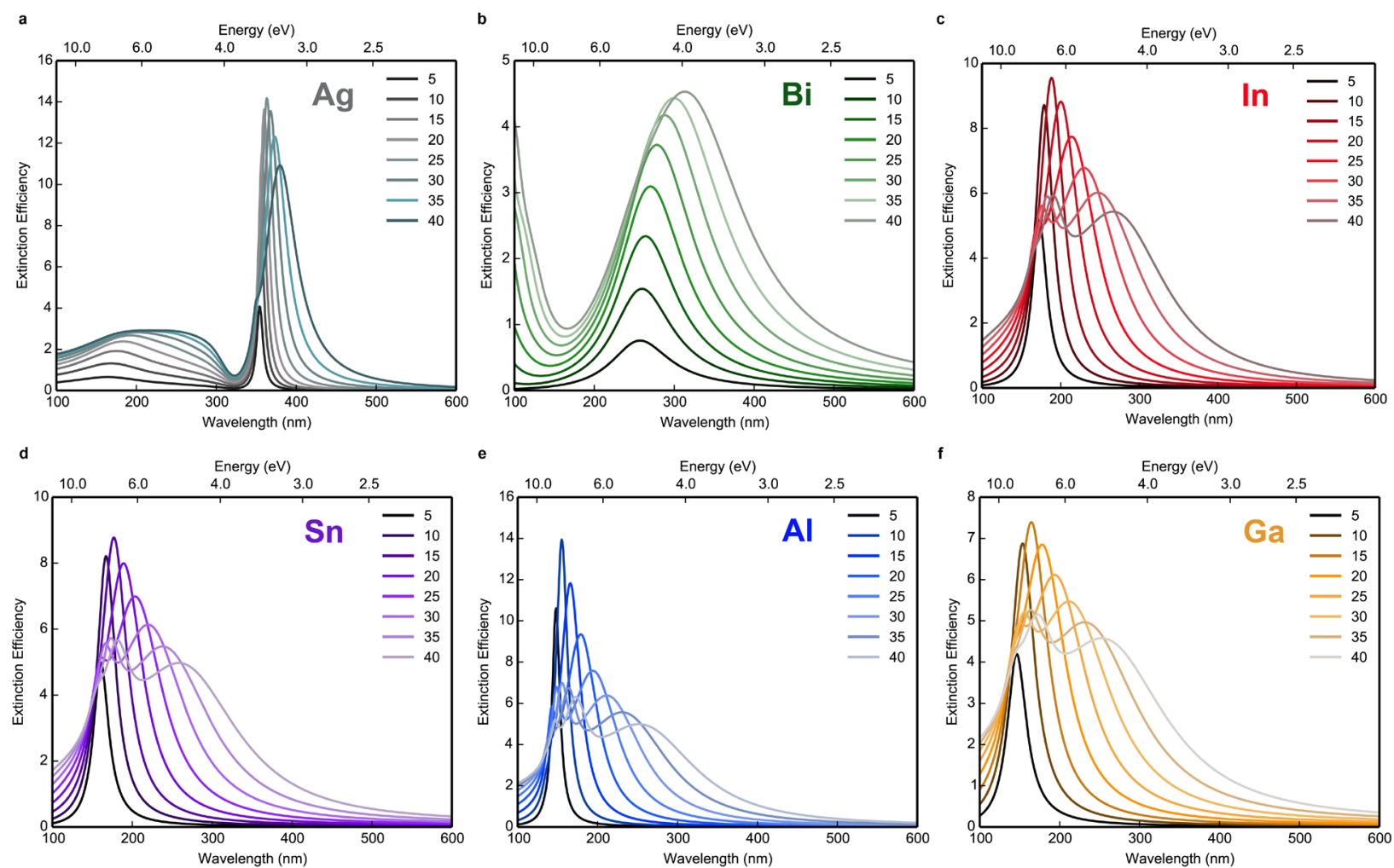

Figure 1. Calculated extinction of Ag, Al, Ga, In, Bi, and Sn spheres. Extinction efficiencies calculated with Mie theory for 5-40 nm radius a) Ag, b) Bi, c) In, d) Sn, e) Al, and f) Ga spheres.

The particular characteristics of the dielectric function have complex physical origins. These characteristics arise from the electronic band structure of the metal and phonon interaction. These interactions can be broken down into three parts, interband transitions which promote the temporary formation of electron-hole pairs, intraband transitions which are related to the plasma frequency, and electron-phonon scattering, which is included in the Drude model as the scattering rate term, $\gamma^{2,10,25}$ The plasma frequency is directly dependent on the free electron density of a metal according to

$$
\omega_{p}=\sqrt{\frac{N e^{2}}{\varepsilon_{0} m_{\mathrm{e}}}}(13)
$$


where $N$ is the number of free carriers, $e$ is the charge of an electron, $\varepsilon_{0}$ is the permittivity of free space, and $m_{\mathrm{e}}$ is the electron mass. The plasma frequency determines the frequencies below which a metal can support a charge oscillation. Thus, while the particular characteristics of the dielectric function determine the complete plasmonic response, the onset of plasmonic activity correlates with the number of free carriers in the metal. In general, the metals with the highest free electron density (Table 1) ${ }^{54}$ have the highest energy LSPRs (Figure 1), though this simple relationship does not account for the important role of interband transitions, as will be discussed below. While this relationship is well known and simplified here, ${ }^{1,10,25}$ it highlights how the intrinsic physical properties of the post-transition metals $\mathrm{Al}, \mathrm{Bi}, \mathrm{Ga}$, In, and $\mathrm{Sn}$ differentiate their plasmonic response from the more conventional noble metals.

Table 1. Electronic properties of metals in increasing order of free electron density.

\begin{tabular}{l|c} 
Metal & $\begin{array}{c}\text { Free Electron Density, } \\
\boldsymbol{n}\left(\mathbf{1 0}^{\mathbf{2 2}} / \mathbf{c m}^{\mathbf{3}}\right)\end{array}$ \\
\hline $\mathrm{Ag}$ & 5.86 \\
$\mathrm{In}$ & 11.5 \\
$\mathrm{Bi}$ & 14.1 \\
$\mathrm{Sn}$ & 14.8 \\
$\mathrm{Ga}$ & 15.4 \\
$\mathrm{Al}$ & 18.1
\end{tabular}


The Mie calculations show that the LSPR redshifts and broadens with an increasing radius for all metals. Additionally, beyond a material-dependent radius, multiple peaks are observed in the extinction spectra. These peaks correspond to higher-order modes e.g. quadrupolar, hexapolar, and octopolar resonances. ${ }^{1,31}$ These emerge due to the depolarization of the incident field across the volume of the particle; they occur when the particle is significantly larger than the wavelength of light, which causes a non-uniform electric field over the surface of the particle. Higher-order modes emerge on the higher energy side of the LSPR peak, for example, the quadrupole for In emerges at $180 \mathrm{~nm}$ for radii of $25 \mathrm{~nm}$, while both a hexapole $(180 \mathrm{~nm})$ and quadrupole $(210 \mathrm{~nm})$ are present for sizes greater than $40 \mathrm{~nm}$. $\mathrm{Al}, \mathrm{Ga}$, and Sn exhibit similar behavior. In contrast, $\mathrm{Ag}$ spheres with radii of 5-35 $\mathrm{nm}$ exhibit only a dipolar mode, with a quadrupole emerging for larger radii. Unlike the other metals, Bi only has a weak quadrupole that emerges at radii greater than $\sim 40 \mathrm{~nm}$.

For comparison with the analytic Mie results, both the quasistatic approximation and MLWA were used to simulate spheres of the same radius (Figures S1 and S2). ${ }^{1,48}$ These two levels of theory account for the size- and wavelength-dependence of the LSPR distinctly and provide useful context for understanding the factors that determine plasmonic response. The quasistatic approximation is generally accurate when the wavelength of radiation is much larger than the particle size; it assumes that the particle is in a spatially uniform electrostatic field. ${ }^{1,48}$ For $\mathrm{Al}, \mathrm{Bi}, \mathrm{Ga}$, In, and $\mathrm{Sn}$, which have LSPRs below $\sim 250 \mathrm{~nm}$, the quasistatic approximation does not correlate well to Mie Theory (Figure S1). This can be rationalized because, for UV light where the LSPR corresponds to a much shorter wavelength, the particle size is much closer to the wavelength. For Ag, the quasistatic approximation is reasonably accurate for spheres with 5-30 
$\mathrm{nm}$ radii while for $\mathrm{Bi}$, spheres remain quasistatic up to a $30 \mathrm{~nm}$ radius. Above these radii, redshifting and broadening of the LSPR is observed (Figure 1).

To account for the red-shifting and broadening observed in the dipolar LSPR, we use MLWA, which includes radiative damping and dynamic depolarization effects (Figure S2). ${ }^{1,29,55}$ Radiative damping is related to the spontaneous emission of photons due to the induced dipole. It has a strong dependence on the free electron density of a metal and the particle volume and it results primarily in damping and broadening. ${ }^{1} 21$ Dynamic depolarization is related to depolarization of the incident radiation across the particle and it results in the red shifting of the LSPR as the particle size increases. Because it can account for these two phenomena, the MLWA accurately describes the LSPR for all materials studied here up to the radius at which higher-order modes emerge (Figure S2). Even for spheres as small as $5 \mathrm{~nm}$ in radius, there is some deviation between the quasistatic approximation and Mie theory for Al, Ga, In, and Sn (Figure S3, S4). Overall, the quasistatic and MLWA calculations suggest that size-dependent effects in the UVactive plasmonic nanoparticle are distinct from those in $\mathrm{Ag}$.

To better understand the measurable quantities that comprise the extinction spectrum, we analyze the absorption and scattering efficiencies. Figure $\mathbf{2}$ depicts the absorption efficiency over the same range of radii as Figure 1. In general, the absorption efficiency is narrower than the extinction and it does not exhibit significant red-shifting with increasing size. For Ag spheres, the absorption efficiency is greater $(\sim 10)$ than that observed for the other metals. While Al has a maximum absorbance efficiency approaching 10 , this occurs for the smallest sphere $(5 \mathrm{~nm})$ and decreases rapidly with increasing size. Similar behavior is observed for Ga, In, and Sn. Previously this size-dependent behavior was ascribed to a red-shifting of the LSPR toward the lossier part of 
the dielectric function for $\mathrm{Al}$, a similar explanation appears to be true for $\mathrm{Ga}$, In, and $\mathrm{Sn} .{ }^{25,}{ }^{31} \mathrm{Bi}$ has the lowest absorption efficiency maximum for any of the metals investigated here.
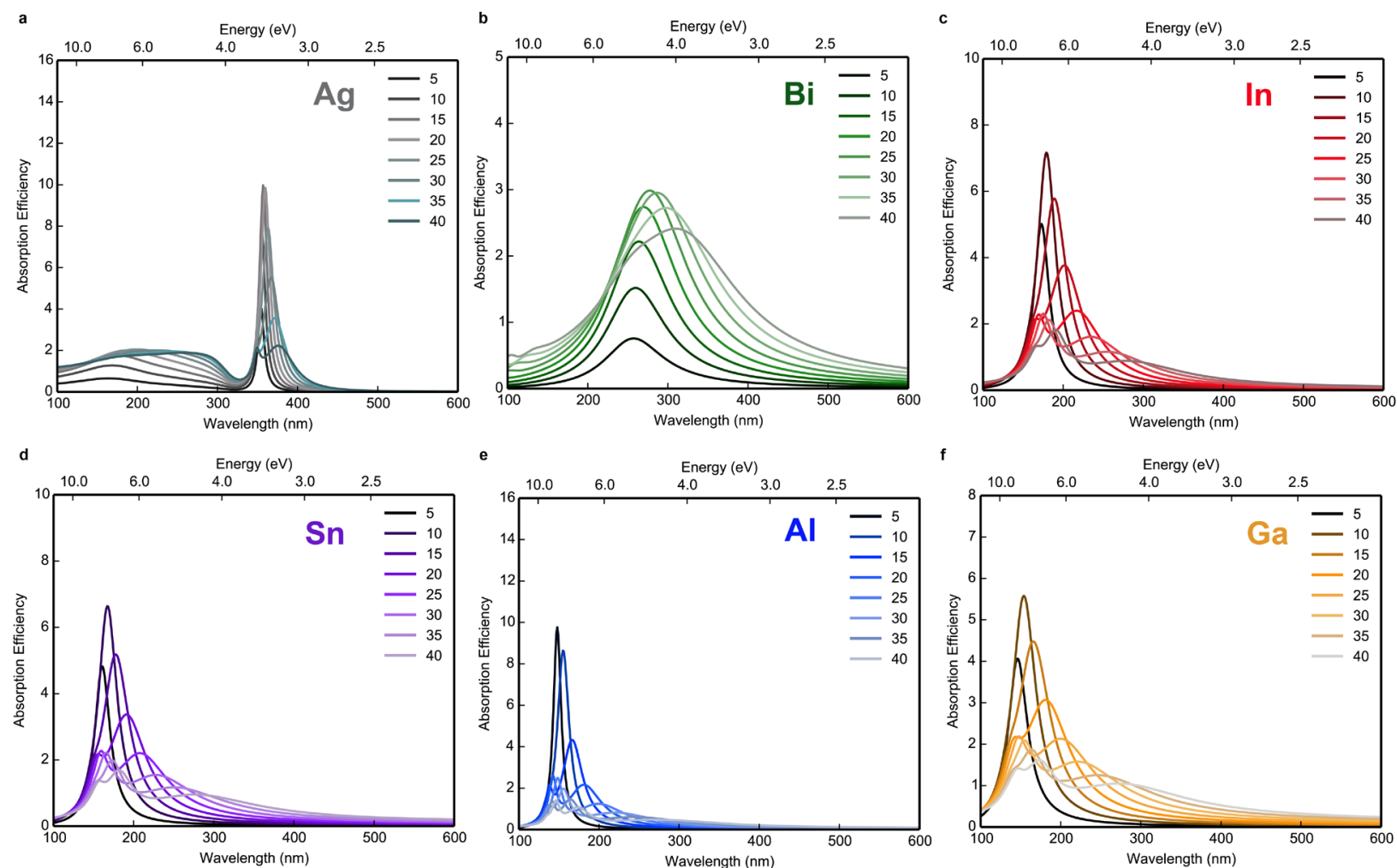

Figure 2. Calculated absorption of Ag, Al, Ga, In, Bi, and Sn spheres. Absorption

efficiencies calculated with Mie theory for 5-40 nm radius a) Ag, b) Bi, c) In, d) Sn, e) Al, and f) Ga spheres.

In comparison with the absorption, the scattering efficiency is broadened, damped, and redshifted (Figure 3). These changes are due to the same phenomena described by the MLWA, namely, radiative damping and dynamic depolarization. ${ }^{1,21}$ As anticipated, Al, Ga, In, and Sn exhibit similar behavior to the extinction spectra, such as broadened LSPRs for sphere radii 
exceeding 20-25 nm. The scattering efficiency exceeds the absorption efficiency in $\mathrm{Al}$ and $\mathrm{Sn}$ for radii greater than $15 \mathrm{~nm}$, in Ga and In for radii greater than $20 \mathrm{~nm}$, in Ag for radii greater than 25 $\mathrm{nm}$, and in $\mathrm{Bi}$ for radii greater than $35 \mathrm{~nm}$. These data suggest that radiative and size-dependent broadening effects are significantly more pronounced in all of the UV-active materials investigated here, such that even at radii as small as $5 \mathrm{~nm}$ they are not well-described by the quasistatic dipole approximation (Figure S3, S4). To better understand the contributions to broadening for the UVresonant metals, it is necessary to investigate the specific linewidth contributions to the LSPR.
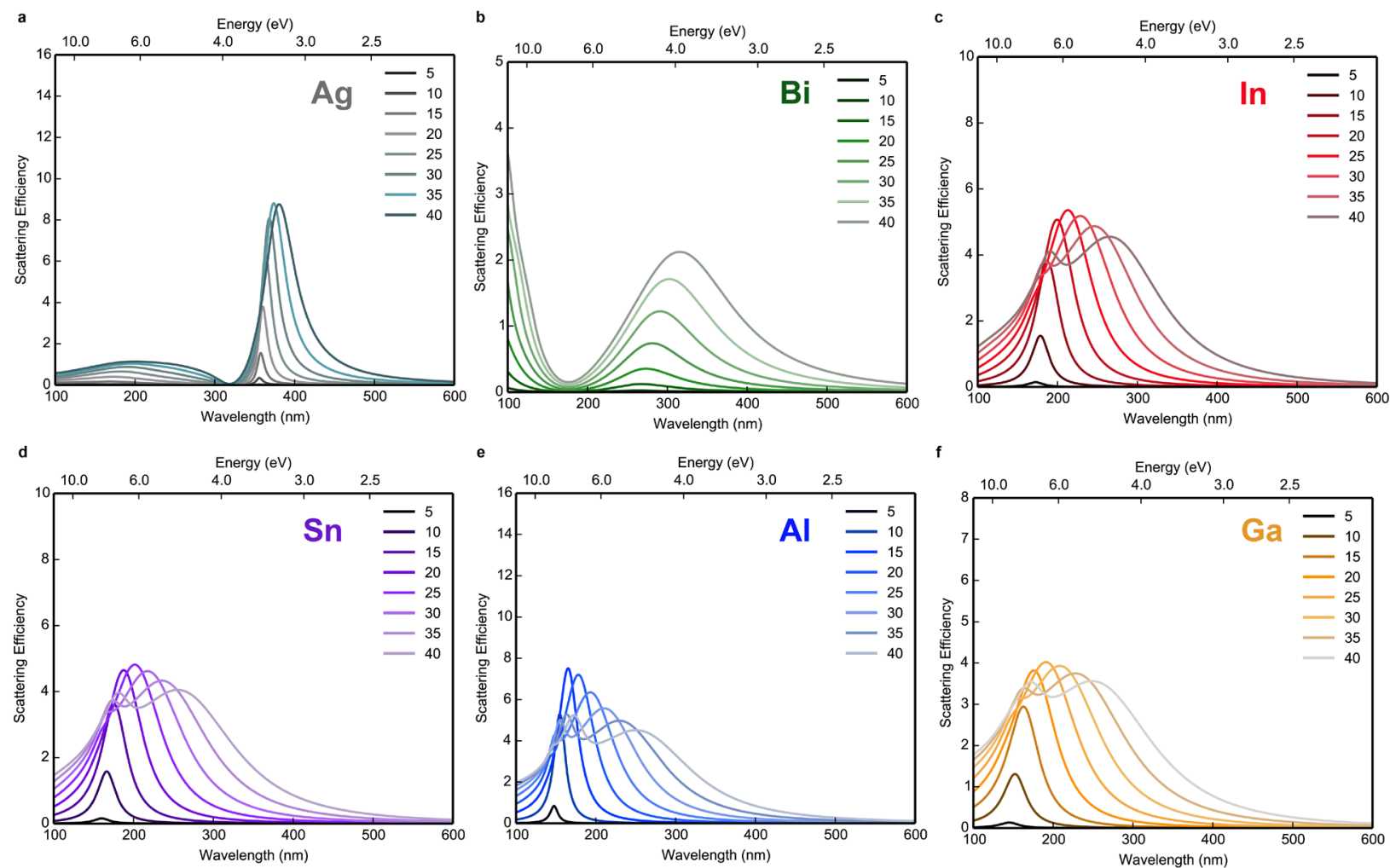

Figure 3. Calculated scattering of $\mathbf{A g}, \mathbf{A l}, \mathbf{G a}, \mathbf{I n}, \mathbf{B i}$, and $\mathrm{Sn}$ spheres. Scattering efficiencies calculated with Mie theory for 5-40 nm radius a) Ag, b) Bi, c) In, d) Sn, e) Al, and f) Ga spheres. 


\section{Contributions to the Linewidth}

The total linewidth is determined by fitting the peak of extinction efficiency and is defined as the full width at half maximum (FWHM) of the dipolar resonance (Methods). Figure 4 shows the size-dependent values of the total linewidth $\Gamma$ that are obtained from the fit of the extinction efficiency. As is well known, Ag has a narrow linewidth, nearly an order of magnitude less than the other metals. For example, for a $15 \mathrm{~nm}$ radius sphere, $\mathrm{Al}$ and $\mathrm{In}$ have linewidths of $\sim 1.3 \mathrm{eV}$, while Ag has a linewidth of $\sim 0.1 \mathrm{eV}$. Similarly, the linewidth is 1.5 and $2.2 \mathrm{eV}$ for $\mathrm{Sn}$ and $\mathrm{Ga}$, respectively. Among the UV active metals, Al exhibits the narrowest linewidth, however, the linewidths for In and $\mathrm{Sn}$ are similar (within $0.3 \mathrm{eV}$ ) for spheres with radii less than $15 \mathrm{~nm}$. 

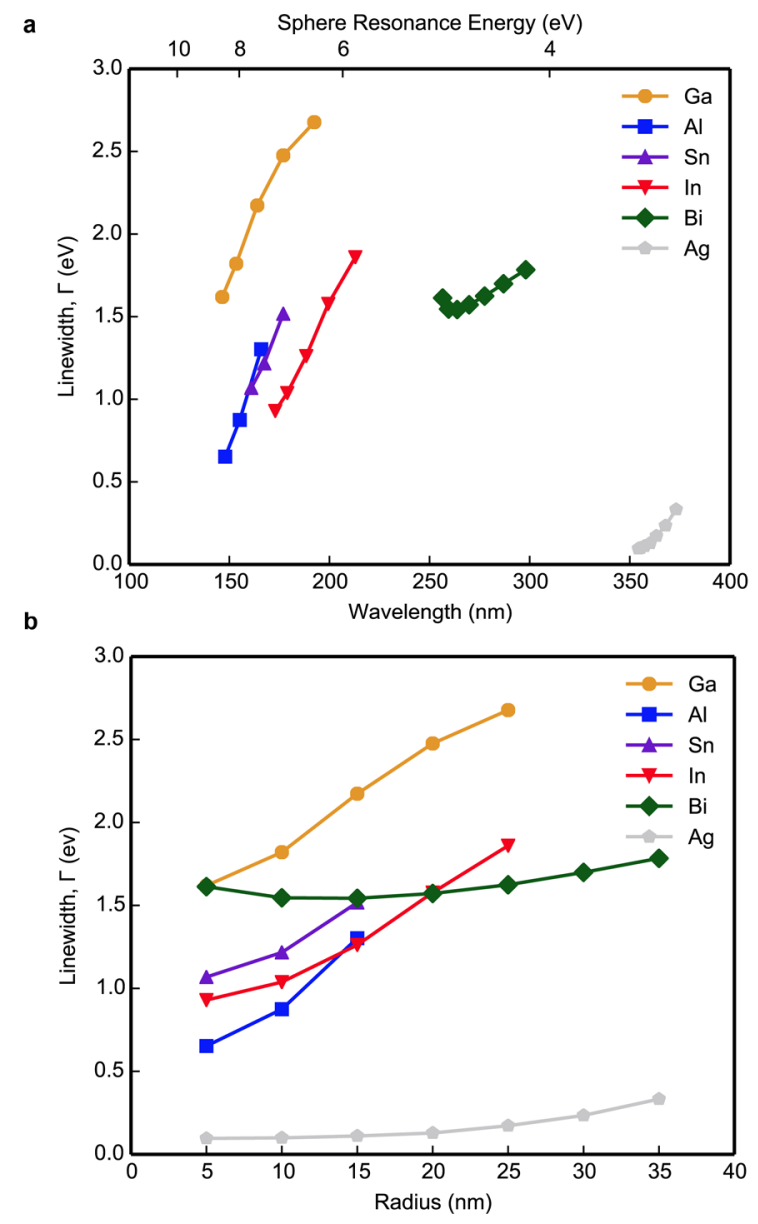

Figure 4. The plasmonic linewidth for Al, Ag, Bi, Ga, In, and Sn spheres. The homogeneous linewidth $\Gamma$ for $\mathrm{Al}$ (blue squares), $\mathrm{Ag}$ (grey pentagons), Bi (green diamonds), Ga (orange circles), In (red nablas), and Sn (purple triangles) as a function of wavelength (a) and radius (b).

To better understand the origin of broadening of the linewidths, it is important to deconvolute the total linewidth into its non-radiative and radiative components (see Methods for more information). ${ }^{19-21}$ Figure 5 depicts the deconvolution of the total linewidth $\Gamma$ for $\mathrm{Ag}, \mathrm{Al}, \mathrm{Bi}$, $\mathrm{Ga}$, In, and Sn spheres. For Ag, the primary contribution to $\Gamma$ is non-radiative up to a radius of 30 $\mathrm{nm}$, above which $\Gamma_{\text {radiative }}$ is a greater contributor. This is because the non-radiative contribution 
does not change significantly as a function of the radius due to the flat nature of the Ag dielectric function over this region while $\Gamma_{\text {radiative }}$ increases as a function of particle volume. ${ }^{19,21,31}$ In contrast, the linewidth contributions for $\mathrm{Al}, \mathrm{Bi}, \mathrm{Ga}, \mathrm{In}$, and $\mathrm{Sn}$ show that $\Gamma$ is primarily composed of radiative broadening, with a small $(<0.01 \mathrm{eV})$ non-radiative contribution. For Al, In, and Sn, $\Gamma_{\text {non-radiative }}$ - the intrinsic broadening due to the bulk dielectric function - is less than $0.01 \mathrm{eV}$, about an order of magnitude smaller than in $\mathrm{Ag}(\sim 0.1 \mathrm{eV})$, while it is $\sim 0.02 \mathrm{eV}$ for $\mathrm{Ga}$. This means that for $\mathrm{Al}, \mathrm{Ga}, \mathrm{In}$, and $\mathrm{Sn}, \Gamma_{\text {radiative }}$ is $\sim 100$-fold greater than $\Gamma_{\text {non-radiative, even for the smallest }}$ sphere radius investigated here $(5 \mathrm{~nm})$.
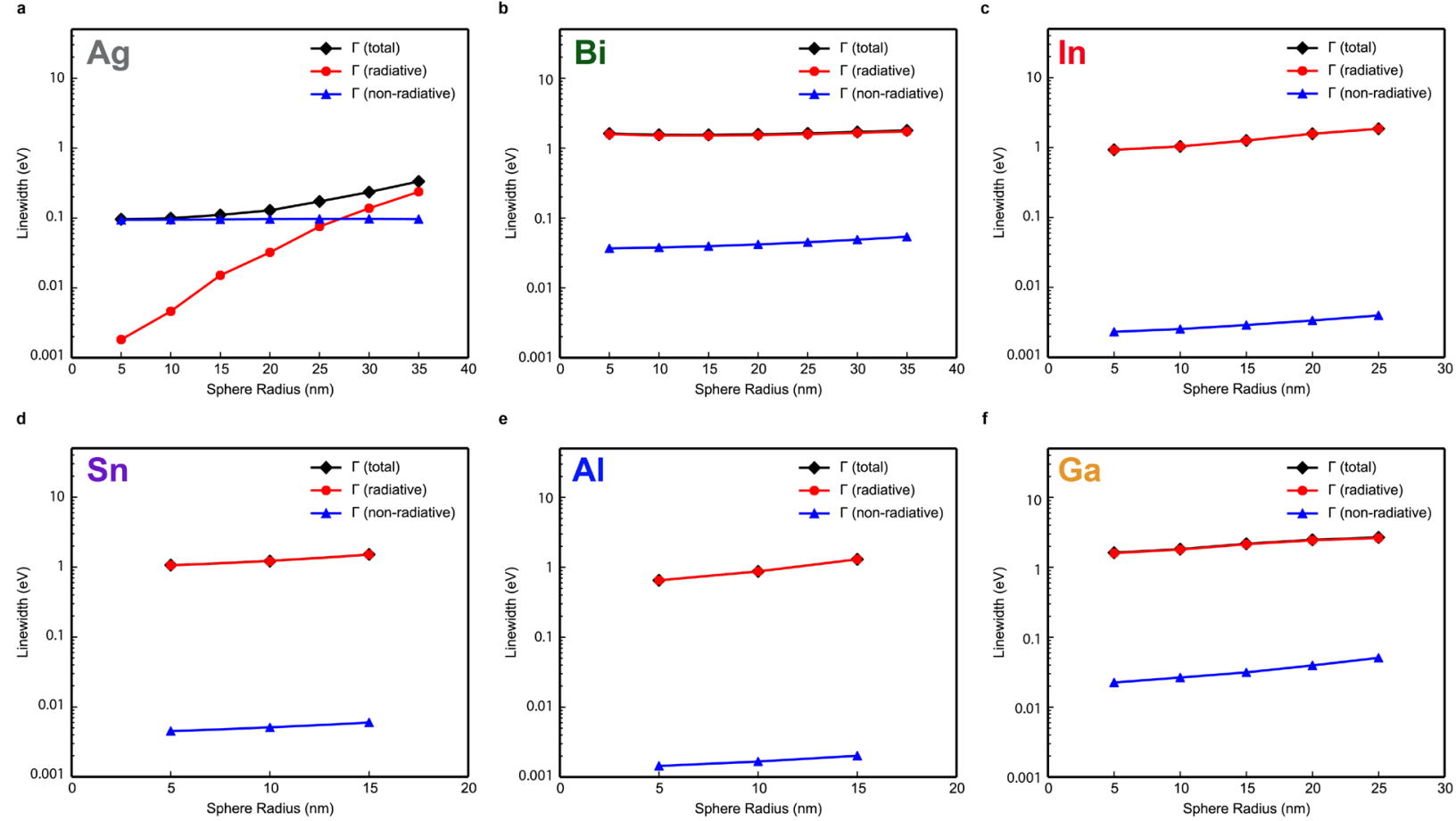

Figure 5. Contributions to the homogeneous linewidth, $\Gamma$. Separation of the total linewidth $\Gamma$ (black diamonds), radiative linewidth $\Gamma_{\text {radiative }}$ (red circles), and $\Gamma_{\text {non-radiative }}$ (blue triangles), for a) $\mathrm{Ag}, \mathrm{b}) \mathrm{Bi}, \mathrm{c}) \mathrm{In}, \mathrm{d}) \mathrm{Sn}, \mathrm{e}) \mathrm{Al}$, and f) Ga. 
We note that the MLWA captures these differences in the broadening contributions (Figure S2) by accounting for dynamic depolarization and radiative damping effects. In particular, this can be understood by the $\mathrm{k}^{3}$ scaling of the radiation damping term in the modified polarizability, which is more pronounced at high-energy LSPRs. ${ }^{31}$ While the linewidth decomposition provides physical insight into the broadening mechanisms for the LSPR, it can also be related to quantities such as the quality factor and plasmon lifetime. ${ }^{20}$

\section{Quality Factors and Dephasing Times}

While the plasmon linewidth and its contributions aid in gaining physical insight into the LSPR for different materials, quantities such as the plasmon quality factor and lifetime are of practical importance and provide context for applications. The quality factor of a resonance, defined as $Q=E_{\mathrm{LSPR}} / \Gamma$, describes the extent of local field enhancement and confinement of light. ${ }^{34,56}$ The highest quality factors are observed for Ag, 10-40, depending on the radius (Figure 6a). However, the Ag LSPR is limited to a small range in the visible region between 360 and 380 nm. In contrast, $\mathrm{Al}, \mathrm{Bi}, \mathrm{Ga}, \mathrm{In}$ and $\mathrm{Sn}$ allow access to LSPRs over the broad range of UV wavelengths from 140-300 nm, depending on the material. However, these LSPRs do have lower quality factors than observed for Ag. For example, Al exhibits quality factors of 6-13 in the range of 150-170 nm, while Sn and In quality factors are in the range of 4-8 in the range of 150-180 nm and 170-230 nm, respectively (Figure 6a).

The homogeneous linewidth was also used to calculate the dephasing times (T) which describe the time scale over which the plasmon oscillation is coherent. ${ }^{20}$ These were determined 
according to the relation $T=2 \hbar / \Gamma .{ }^{19,50}$ The dephasing time describes the time scale over which a particle can support a coherent oscillation, where decoherence occurs due to radiation damping and non-radiative processes. In general, the LSPR dephasing time increases with size due to the effect of increasing particle volume on broadening (Figure 6b). ${ }^{20-21}$ As expected, Ag shows the longest dephasing time of $14 \mathrm{fs}$ for a sphere with a $5 \mathrm{~nm}$ radius. By comparison, the dephasing times for $\mathrm{Al}, \mathrm{Ga}$, In, and $\mathrm{Sn}$ are in the range from 1-2.5 fs, while $\mathrm{Ga}$ and $\mathrm{Bi}$ are both less than $1 \mathrm{fs}$ for all size spheres studied here.

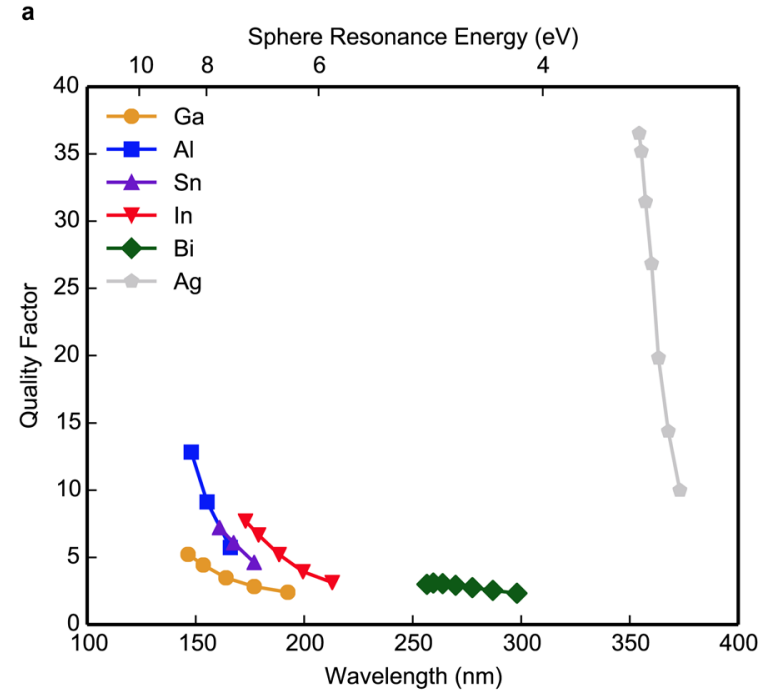

b

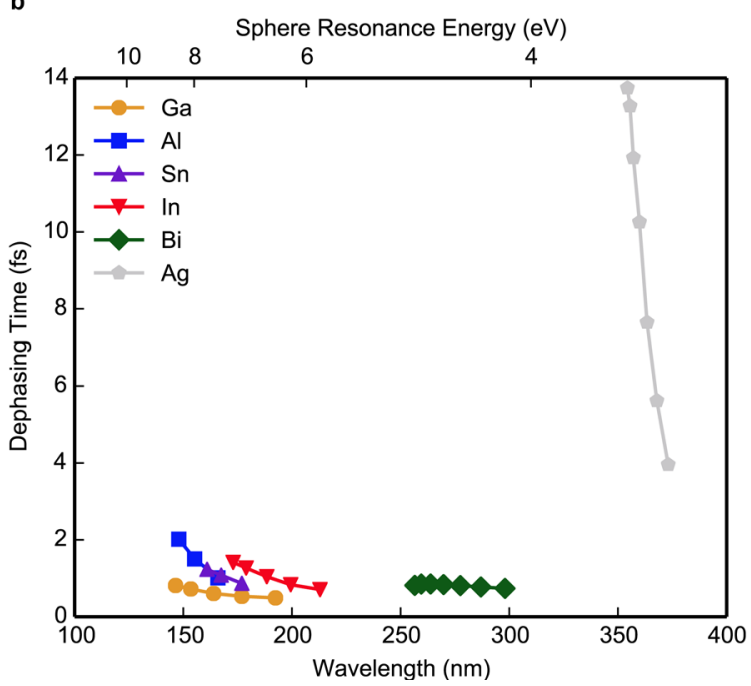


Figure 6. The plasmonic quality and lifetime. The calculated plasmon quality factor a) and lifetime (b) for Ga (orange circles), Al (blue squares), Sn (purple triangles), In (red nablas), Bi (green diamonds), and Ag (grey pentagons).

Generalized Analysis of Linewidth Broadening Using An Ideal Free Electron Model

For a more generalized and physical interpretation of the impact of the LSPR energy on the linewidth, we use a Drude model to generate dielectric functions for ideal free-electron metals with plasma frequencies from 6-20 eV (Figure S5) ${ }^{52-53}$ We note that this model includes minimal losses in the imaginary part of the dielectric function as compared to what is observed in the metals discussed above (Figure S5). Figure 7a depicts the extinction efficiencies for the free-electron Drude metals calculated using Mie theory for a $10 \mathrm{~nm}$ radius sphere. It is observed that the extinction maxima occur at higher energies for increasing plasma frequency metals. To more quantitatively understand the relation between plasma frequency and linewidth, Figure 7b shows the linewidth analysis for the ideal free-electron Drude metal Mie calculations. Here, increasing plasma frequency metals exhibit increasingly broad LSPRs. 
a
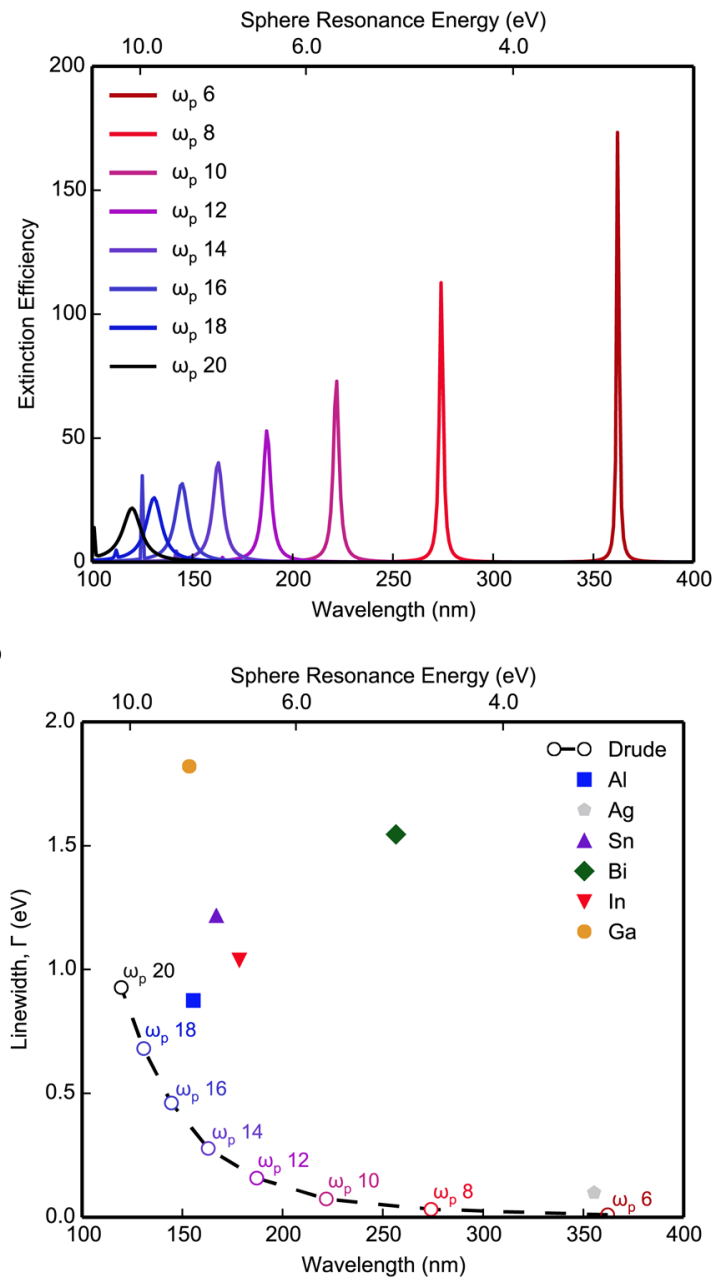

C

$\mathrm{Ga}, \mathrm{Al}, \mathrm{Sn}, \mathrm{In} \quad \mathrm{Ag}$

$150 \mathrm{~nm}$ $400 \mathrm{~nm}$

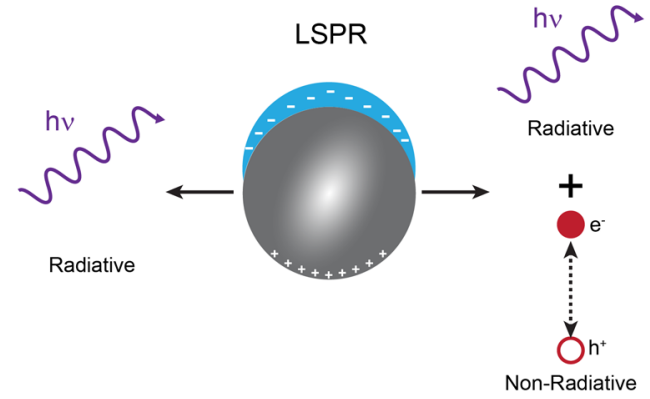

Figure 7. Model for ideal free-electron Drude metals.a) The calculated extinction spectra for ideal free-electron Drude metals with plasma frequencies ranging from 6-20 eV for a $10 \mathrm{~nm}$ radius sphere. (b) Homogeneous linewidth $\Gamma$ as a function of sphere resonance energy and wavelength 
for the ideal Drude metals and for real metals for a $10 \mathrm{~nm}$ radius sphere: $\mathrm{Al}$ (blue square), $\mathrm{Ag}$ (grey pentagon), Bi (green diamond), Ga (orange circle), In (red nabla), and Sn (purple triangle). c) Depicts the primary linewidth contributions and wavelengths for the plasmonic materials investigated in this work.

For comparison, we include the linewidths for $\mathrm{Al}, \mathrm{Ag}, \mathrm{Bi}, \mathrm{Ga}$, In, and $\mathrm{Sn}$ spheres of the same size (Figure 4). Overall, we see that these metals do not follow a systematic trend in comparison with the ideal Drude metals. This is due to the unique dielectric function (Figure S5) of each metal, which adds significant complexity in comparison with the simple idealized Drude model. However, excluding Bi and Ga, which can have complex interband-driven plasmons, ${ }^{57}$ it is seen that the linewidths are comparatively greater for higher energy plasmons than for $\mathrm{Ag}$ plasmons.

It remains difficult to generalize these results for all of the plasmonic metals due to the unique intrinsic behavior that influences the plasmon linewidth and LSPR energy. Even comparing metals such as $\mathrm{Ga}$ and In that have similar plasma frequencies $(\sim 1 \mathrm{eV}$ difference), it is clear that the interband behavior greatly impacts the extinction behavior near the resonance energy (Table S1). Bi and Ga exhibit interband plasmonic behavior that differentiates them from traditional plasmonic metals, and they deviate more from the plasma frequency dependence exhibited by the ideal free-electron metals. ${ }^{57}$ In and Sn exhibit similar plasmon broadening, have similar real and imaginary parts of their dielectric functions (Figure S5), and lie closer to the ideal free-electron Drude metals. ${ }^{25}$ Finally, $\mathrm{Ag}$ and $\mathrm{Al}$ exhibit the most Drude-like behavior in the real part of the 
dielectric function, and their linewidths are most closely approximated by the ideal free-electron Drude model (Figure 7b).

When taken together, we show the trend of increased plasmon broadening for higher energy plasmons is observed for ideal free-electron Drude metals. However, the distinct interband contributions and dielectric behavior for each metal prevents a generalizable analysis (Figure S5, Table S1). These data show the impact of the high resonance energy on the large radiative contribution to broadening (Figure 7c) in Al, Ga, In, and Sn, while Ag, with a lower energy LSPR, is not susceptible to the same extent of radiative broadening, hence the total linewidth is made up of non-radiative and radiative contributions.

\section{Conclusion}

We have calculated the optical properties of spherical nanoparticles for metals with LSPRs throughout the UV. We find that radiative contributions dominate the plasmonic response for $\mathrm{Al}$, $\mathrm{Ga}, \mathrm{In}, \mathrm{Sn}$, and, to a lesser extent $\mathrm{Bi}$, when compared to Ag. These results are consistent with previous investigations for $\mathrm{Al}$ and noble metal nanoparticles $\mathrm{s}^{20-21,31-32,56}$ while demonstrating the significance of radiative effects for promising UV-active post-transition plasmonic metals (Figure 7c). For the radii studied here $(5-40 \mathrm{~nm})$, the radiative contribution makes up a larger fraction than the non-radiative portion of the linewidth even for spheres as small as $5 \mathrm{~nm}$ in radius. This is distinct from the behavior of $\mathrm{Au}$ and $\mathrm{Ag}$, where non-radiative contributions dictate the linewidth for small spheres. ${ }^{10,20,31}$ While there are relatively few experimental investigations for small Ga, In, and Sn spheres, the calculated optical spectra and quality factors for these metals suggest that their behavior would be qualitatively similar to $\mathrm{Al}$ in the UV. Finally, the deviations in broadening 
from metal to metal arise from a combination of the location of the LSPR and the intrinsic dielectric behavior of the metal. ${ }^{57}$

These results have broad implications for designing functional plasmonic nanoparticles in the UV. The relative significance of radiative effects in small Al, Ga, In, and Sn spheres suggests that radiative damping and scattering contributions could be a challenge for plasmonic nanoparticles in the deep UV, however, there is evidence that Au dimers can still provide Raman enhancement with significant radiative effects. ${ }^{58}$ Furthermore, there are demonstrations that anisotropic or film-coupled Al nanoparticles can support high-quality LSPRs and overcome radiation damping pathways, similar possibilities likely exist for the other post-transition metals. 32, 34, 59-60 Meanwhile, strategies that involve the arrangement of plasmonic nanoparticles into twodimensional ordered arrays can dramatically narrow linewidths and manipulate radiative effects in lasing arrays, metasurfaces, and pixel-based devices; similar strategies should be translatable to the metals discussed herein. ${ }^{7,61-62}$ This work demonstrates that the plasmon resonances for the post-transition metals $\mathrm{Al}, \mathrm{Bi}, \mathrm{Ga}, \mathrm{In}$ and $\mathrm{Sn}$ in the $\mathrm{UV}$ are distinct from those for noble metal nanoparticles and that these materials have distinct optical behavior in the UV that provides new opportunities for plasmonics.

\section{Supporting Information}

Quasistatic and modified-long wavelength approximation calculated spectra, dielectric functions, and plasma frequencies for $\mathrm{Ag}, \mathrm{Al}, \mathrm{Bi}, \mathrm{Ga}, \mathrm{In}$, and $\mathrm{Sn}$ and for ideal Drude metals are included.

The Supporting Information is available free of charge on the ACS Publications website. 


\section{Author Information:}

Corresponding Author:

Correspondence and requests for materials should be sent to M.B.R. (Michael_ross@uml.edu).

ORCID:

Michael B. Ross 0000-0002-2511-0594

Maria V. Fonseca Guzman 0000-0001-8931-1224

\section{Author Contributions}

All authors have given approval to the final version of the manuscript.

\section{Conflicts of Interest}

Notes:

The authors declare no competing financial interests.

\section{Acknowledgments}

This material was supported by the University of Massachusetts Lowell and the Commonwealth of Massachusetts. M.V.F.G. gratefully acknowledges support from the RIST Institute for Sustainability and Energy. 


\section{References}

1. Kelly, K. L.; Coronado, E.; Zhao, L. L.; Schatz, G. C., The Optical Properties of Metal Nanoparticles: The Influence of Size, Shape, and Dielectric Environment. J. Phys. Chem. B 2003, 107, 668-677.

2. $\quad$ Kreibig, U.; Vollmer, M., Optical Properties of Metal Clusters; Springer: Berlin, 1995.

3. Quinten, M., Optical Properties of Nanoparticle Systems; Wiley-VCH: Weinheim, 2011.

4. $\quad$ Ben-Jaber, S.; Peveler, W. J.; Quesada-Cabrera, R.; Cortés, E.; Sotelo-Vazquez, C.; Abdul-

Karim, N.; Maier, S. A.; Parkin, I. P., Photo-Induced Enhanced Raman Spectroscopy for Universal Ultra-Trace Detection of Explosives, Pollutants and Biomolecules. Nat. Commun. 2016, 7.

5. Brongersma, M. L.; Halas, N. J.; Nordlander, P., Plasmon-Induced Hot Carrier Science and Technology. Nat. Nanotechnol 2015, 10, 25-34.

6. Langer, J., et al., Present and Future of Surface-Enhanced Raman Scattering. ACS Nano 2019, 14, 28-117.

7. Wang, D.; Guan, J.; Hu, J.; Bourgeois, M. R.; Odom, T. W., Manipulating Light-Matter Interactions in Plasmonic Nanoparticle Lattices. Acc. Chem. Res. 2019, 52, 2997-3007.

8. Ross, M. B.; Mirkin, C. A.; Schatz, G. C., Optical Properties of One-, Two-, and ThreeDimensional Arrays of Plasmonic Nanostructures. J. Phys. Chem. C 2016, 120, 816-830.

9. Jones, M. R.; Osberg, K. D.; Macfarlane, R. J.; Langille, M. R.; Mirkin, C. A., Templated Techniques for the Synthesis and Assembly of Plasmonic Nanostructures. Chem. Rev. 2011, 111, 3736-3827.

10. Blaber, M. G.; Arnold, M. D.; Ford, M. J., A Review of the Optical Properties of Alloys and Intermetallics for Plasmonics. J. Phys.: Condens. Matter 2010, 22.

11. West, P. R.; Ishii, S.; Naik, G. V.; Emani, N. K.; Shalaev, V. M.; Boltasseva, A., Searching for Better Plasmonic Materials. Laser Photonics Rev. 2010, 4, 795-808.

12. Kanipe, K. N.; Chidester, P. P. F.; Stucky, G. D.; Meinhart, C. D.; Moskovits, M., Properly Structured, Any Metal Can Produce Intense Surface Enhanced Raman Spectra. J. Phys. Chem. C 2017, 121, 14269-14273.

13. Bezerra, A. G.; Machado, T. N.; Woiski, T. D.; Turchetti, D. A.; Lenz, J. A.; Akcelrud, L.; Schreiner, W. H., Plasmonics and SERS Activity of Post-Transition Metal Nanoparticles. J. Nanoparticle Res. 2018, 20.

14. Lin, B. J., Deep UV Lithography. Journal of Vacuum Science and Technology 1975, 12, 1317-1320.

15. Barulin, A.; Claude, J.-B.; Patra, S.; Bonod, N.; Wenger, J., Deep Ultraviolet Plasmonic Enhancement of Single Protein Autofluorescence in Zero-Mode Waveguides. Nano Lett. 2019, 19, 7434-7442.

16. Chowdhury, M. H.; Ray, K.; Gray, S. K.; Pond, J.; Lakowicz, J. R., Aluminum Nanoparticles as Substrates for Metal-Enhanced Fluorescence in the Ultraviolet for the Label-Free Detection of Biomolecules. Anal. Chem. 2009, 81, 1397-1403.

17. Sharma, B.; Cardinal, M. F.; Ross, M. B.; Zrimsek, A. B.; Bykov, S. V.; Punihaole, D.; Asher, S. A.; Schatz, G. C.; Van Duyne, R. P., Aluminum Film-over-Nanosphere Substrates for Deep-UV Surface-Enhanced Resonance Raman Spectroscopy. Nano Lett. 2016, 16, 7968-7973. 
18. Jha, S. K.; Ahmed, Z.; Agio, M.; Ekinci, Y.; Löffler, J. F., Deep-UV Surface-Enhanced Resonance Raman Scattering of Adenine on Aluminum Nanoparticle Arrays. J. Am. Chem. Soc. 2012, 134, 1966-1969.

19. Blaber, M. G.; Henry, A.-I.; Bingham, J. M.; Schatz, G. C.; Van Duyne, R. P., Lspr Imaging of Silver Triangular Nanoprisms: Correlating Scattering with Structure Using Electrodynamics for Plasmon Lifetime Analysis. J. Phys. Chem. C 2011, 116, 393-403.

20. Hartland, G. V., Optical Studies of Dynamics in Noble Metal Nanostructures. Chem. Rev. 2011, 111, 3858-3887.

21. Zorić, I.; Zäch, M.; Kasemo, B.; Langhammer, C., Gold, Platinum, and Aluminum Nanodisk Plasmons: Material Independence, Subradiance, and Damping Mechanisms. ACS Nano 2011, 5, 2535-2546.

22. Gutierrez, Y.; Alcaraz de la Osa, A.; Ortiz, D.; Saiz, J. M.; Gonzalez, F.; Moreno, F., Plasmonics in the Ultraviolet with Aluminum, Gallium, Magnesium and Rhodium. Appl. Sci. 2018, 8 .

23. Biggins, J. S.; Yazdi, S.; Ringe, E., Magnesium Nanoparticle Plasmonics. Nano Lett. 2018, $18,3752-3758$.

24. Knight, M. W.; King, N. S.; Liu, L.; Everitt, H. O.; Nordlander, P.; Halas, N. J., Aluminum for Plasmonics. ACS Nano 2013, 8, 834-840.

25. McMahon, J. M.; Schatz, G. C.; Gray, S. K., Plasmonics in the Ultraviolet with the Poor Metals Al, Ga, in, Sn, Tl, Pb, and Bi. Phys. Chem. Chem. Phys. 2013, 15, 5415-5423.

26. Toudert, J.; Serna, R.; Camps, I.; Wojcik, J.; Mascher, P.; Rebollar, E.; Ezquerra, T. A., Unveiling the Far Infrared-to-Ultraviolet Optical Properties of Bismuth for Applications in Plasmonics and Nanophotonics. J. Phys. Chem. C 2017, 121, 3511-3521.

27. Watson, A. M.; Zhang, X.; Alcaraz de la Osa, R.; Sanz, J. M.; González, F.; Moreno, F.; Finkelstein, G.; Liu, J.; Everitt, H. O., Rhodium Nanoparticles for Ultraviolet Plasmonics. Nano Lett. 2015, 15, 1095-1100.

28. Yang, Y.; Callahan, J. M.; Kim, T.-H.; Brown, A. S.; Everitt, H. O., Ultraviolet Nanoplasmonics: A Demonstration of Surface-Enhanced Raman Spectroscopy, Fluorescence, and Photodegradation Using Gallium Nanoparticles. Nano Lett. 2013, 13, 2837-2841.

29. Zeman, E. J.; Schatz, G. C., An Accurate Electromagnetic Theory Study of Surface Enhancement Factors for Silver, Gold, Copper, Lithium, Sodium, Aluminum, Gallium, Indium, Zinc, and Cadmium. J. Phys. Chem. 2002, 91, 634-643.

30. Ostovar, B., et al., Acoustic Vibrations of Al Nanocrystals: Size, Shape, and Crystallinity Revealed by Single-Particle Transient Extinction Spectroscopy. J. Phys. Chem. A 2020, 124, 39243934.

31. Ross, M. B.; Schatz, G. C., Radiative Effects in Plasmonic Aluminum and Silver Nanospheres and Nanorods. J. Phys. D: Appl. Phys. 2015, 48.

32. Sobhani, A.; Manjavacas, A.; Cao, Y.; McClain, M. J.; García de Abajo, F. J.; Nordlander, P.; Halas, N. J., Pronounced Linewidth Narrowing of an Aluminum Nanoparticle Plasmon Resonance by Interaction with an Aluminum Metallic Film. Nano Lett. 2015, 15, 6946-6951.

33. Su, M.-N., et al., Ultrafast Electron Dynamics in Single Aluminum Nanostructures. Nano Lett. 2019, 19, 3091-3097.

34. Zhu, X.; Imran Hossain, G. M.; George, M.; Farhang, A.; Cicek, A.; Yanik, A. A., Beyond Noble Metals: High Q-Factor Aluminum Nanoplasmonics. ACS Photonics 2020, 7, 416-424. 
35. Cuadrado, A.; Toudert, J.; Serna, R., Polaritonic-to-Plasmonic Transition in Optically Resonant Bismuth Nanospheres for High-Contrast Switchable Ultraviolet Meta-Filters. IEEE Photonics J. 2016, 8, 1-11.

36. Foerster, B.; Spata, V. A.; Carter, E. A.; Sönnichsen, C.; Link, S., Plasmon Damping Depends on the Chemical Nature of the Nanoparticle Interface. Sci. Adv. 2019, 5.

37. Januar, M.; Liu, B.; Cheng, J.-C.; Hatanaka, K.; Misawa, H.; Hsiao, H.-H.; Liu, K.-C., Role of Depolarization Factors in the Evolution of a Dipolar Plasmonic Spectral Line in the Far- and near-Field Regimes. J. Phys. Chem. C 2020, 124, 3250-3259.

38. Sönnichsen, C.; Franzl, T.; Wilk, T.; von Plessen, G.; Feldmann, J.; Wilson, O.; Mulvaney, P., Drastic Reduction of Plasmon Damping in Gold Nanorods. Phys. Rev. Lett. 2002, 88.

39. Johnson, P. B.; Christy, R. W., Optical Constants of the Noble Metals. Phys. Rev. B 1972, 6, 4370-4379.

40. Palik, E. D.; Ghosh, G., Handbook of Optical Constants of Solids; Academic: Oralndo, FL, 1985.

41. de Sande, J. C. G.; Missana, T.; Afonso, C. N., Optical Properties of Pulsed Laser Deposited Bismuth Films. J. Appl. Phys. 1996, 80, 7023-7027.

42. Hunderi, O.; Ryberg, R., Band Structure and Optical Properties of Gallium. J. Phys. F: Met. Phys. 1974, 4, 2084-2095.

43. Jezequel, G.; Lemonnier, J. C.; Thomas, J., Optical Properties of Gallium Films between 2 and 15 Ev. J. Phys. F: Met. Phys. 1977, 7, 1613-1622.

44. Koyama, R. Y.; Smith, N. V.; Spicer, W. E., Optical Properties of Indium. Physical Review $B$ 1973, 8, 2426-2432.

45. MacRae, R. A.; Arakawa, E. T.; Williams, M. W., Optical Properties of VacuumEvaporated White Tin. Phys. Rev. 1967, 162, 615-620.

46. Linic, S.; Chavez, S.; Elias, R., Flow and Extraction of Energy and Charge Carriers in Hybrid Plasmonic Nanostructures. Nat. Mater. 2021.

47. Mie, G., Beiträge Zur Optik Trüber Medien, Speziell Kolloidaler Metallösungen. Ann. Phys. 1908, 330, 377-445.

48. Bohren, C. F.; Huffman, D. R., Absorption and Scattering of Light by Small Particles; Wiley: New York, 1983.

49. Fan, X.; Zheng, W.; Singh, D. J., Light Scattering and Surface Plasmons on Small Spherical Particles. Light Sci. Appl. 2014, 3, e179-e179.

50. Bosbach, J.; Hendrich, C.; Stietz, F.; Vartanyan, T.; Träger, F., Ultrafast Dephasing of Surface Plasmon Excitation in Silver Nanoparticles: Influence of Particle Size, Shape, and Chemical Surrounding. Phys. Rev. Lett. 2002, 89.

51. Kreibig, U., Small Silver Particles in Photosensitive Glass: Their Nucleation and Growth. Appl. Phys. 1976, 10, 255-264.

52. Pellarin, M.; Broyer, M.; Lermé, J.; Lebeault, M.-A.; Ramade, J.; Cottancin, E., Plasmon Resonances Tailored by Fano Profiles in Silver-Based Core-Shell Nanoparticles. Phys. Chem. Chem. Phys. 2016, 18, 4121-4133.

53. Wang, F.; Shen, Y. R., General Properties of Local Plasmons in Metal Nanostructures. Phys. Rev. Lett. 2006, 97.

54. Ashcroft, N.W.; Mermin, N. D., Solid State Physics; Saunders College Publishing: New York, 1976. 
55. Rasskazov, I. L.; Zakomirnyi, V. I.; Utyushev, A. D.; Carney, P. S.; Moroz, A., Remarkable Predictive Power of the Modified Long Wavelength Approximation. J. Phys. Chem. C 2021, 125, 1963-1971.

56. Sanz, J. M.; Ortiz, D.; Alcaraz de la Osa, R.; Saiz, J. M.; González, F.; Brown, A. S.; Losurdo, M.; Everitt, H. O.; Moreno, F., Uv Plasmonic Behavior of Various Metal Nanoparticles in the near- and Far-Field Regimes: Geometry and Substrate Effects. J. Phys. Chem. C 2013, 117, 19606-19615.

57. Toudert, J.; Serna, R., Ultraviolet-Visible Interband Plasmonics with P-Block Elements. Opt. Mater. Express 2016, 6.

58. Blaber, M. G.; Schatz, G. C., Extending SERS into the Infrared with Gold Nanosphere Dimers. Chem. Commun. 2011, 47.

59. Chan, G. H.; Zhao, J.; Schatz, G. C.; Van Duyne, R. P., Localized Surface Plasmon Resonance Spectroscopy of Triangular Aluminum Nanoparticles. J. Phys. Chem. C 2008, 112, 13958-13963.

60. $\quad$ Knight, M. W.; Liu, L.; Wang, Y.; Brown, L.; Mukherjee, S.; King, N. S.; Everitt, H. O.; Nordlander, P.; Halas, N. J., Aluminum Plasmonic Nanoantennas. Nano Lett. 2012, 12, 6000-6004. 61. Yang, A.; Hryn, A. J.; Bourgeois, M. R.; Lee, W.-K.; Hu, J.; Schatz, G. C.; Odom, T. W., Programmable and Reversible Plasmon Mode Engineering. Proc. Natl. Acad. Sci. U.S.A. 2016, $113,14201-14206$.

62. Olson, J.; Manjavacas, A.; Liu, L.; Chang, W.-S.; Foerster, B.; King, N. S.; Knight, M. W.; Nordlander, P.; Halas, N. J.; Link, S., Vivid, Full-Color Aluminum Plasmonic Pixels. Proc. Natl. Acad. Sci. U.S.A. 2014, 111, 14348-14353.

\section{TOC Image}

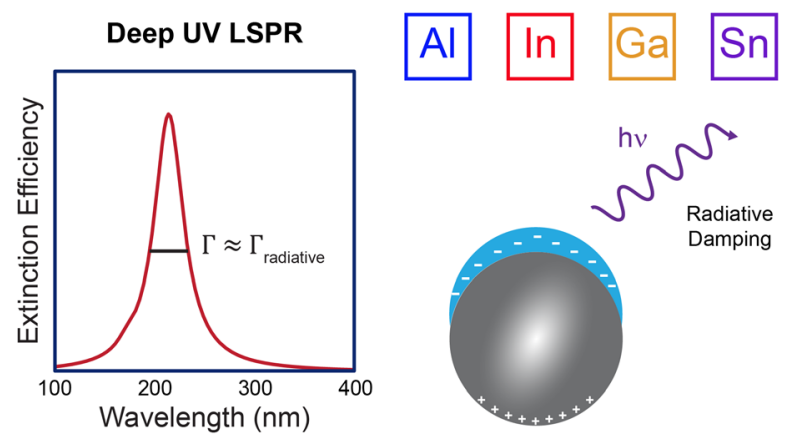

